\title{
Sub-Poissonian Shot Noise in Graphene
}

\author{
J. Tworzydło, ${ }^{1}$ B. Trauzettel, ${ }^{2}$ M. Titov, ${ }^{3}$ A. Rycerz, ${ }^{2,4}$ and C. W. J. Beenakker ${ }^{2}$ \\ ${ }^{1}$ Institute of Theoretical Physics, Warsaw University, Hoża 69, 00-681 Warsaw, Poland \\ ${ }^{2}$ Instituut-Lorentz, Universiteit Leiden, P.O. Box 9506, 2300 RA Leiden, The Netherlands \\ ${ }^{3}$ Department of Physics, Konstanz University, D-78457 Konstanz, Germany \\ ${ }^{4}$ Marian Smoluchowski Institute of Physics, Jagiellonian University, Kraków, Poland
}

(Received 29 March 2006; published 20 June 2006)

\begin{abstract}
We calculate the mode-dependent transmission probability of massless Dirac fermions through an ideal strip of graphene (length $L$, width $W$, no impurities or defects) to obtain the conductance and shot noise as a function of Fermi energy. We find that the minimum conductivity of order $e^{2} / h$ at the Dirac point (when the electron and hole excitations are degenerate) is associated with a maximum of the Fano factor (the ratio of noise power and mean current). For short and wide graphene strips the Fano factor at the Dirac point equals 1/3, 3 times smaller than for a Poisson process. This is the same value as for a disordered metal, which is remarkable since the classical dynamics of the Dirac fermions is ballistic.
\end{abstract}

PACS numbers: 73.50.Td, 73.23.Ad, 73.63.-b

Two recent experiments [1,2] have discovered that the conductivity of graphene (a single atomic layer of carbon) tends to a minimum value of the order of the quantum unit $e^{2} / h$ when the concentration of charge carriers tends to zero. This quantum-limited conductivity is an intrinsic property of two-dimensional Dirac fermions (massless excitations governed by a relativistic wave equation), which persists in an ideal crystal without any impurities or lattice defects [3-6]. In the absence of impurity scattering, and at zero temperature, one might expect the electrical current to be noiseless. In contrast, we show that the minimum in the conductivity is associated with a maximum in the Fano factor (the ratio of noise power and mean current). The Fano factor at zero carrier concentration takes on the universal value $1 / 3$ for a short and wide graphene strip. This is 3 times smaller than the Poissonian noise in a tunnel junction and identical to the value in a disordered metal $[7,8]$ - even though the classical dynamics in the graphene strip is ballistic.

Shot noise measurements have proven to be a valuable diagnostic tool in carbon nanotubes, which can be thought of as rolled-up sheets of graphene. Very low shot noise in well-contacted bundles of single-wall nanotubes is an indication of nearly ballistic one-dimensional transport [9]. Super-Poissonian noise has been found in a quantum dot formed out of a single-wall nanotube, and explained in terms of inelastic tunneling in this zero-dimensional system [10]. Our prediction of sub-Poissonian shot noise in two-dimensional graphene is another manifestation of the importance of dimensionality for quantum transport.

Our analysis of the shot noise was inspired by an insightful recent paper of Katsnelson [6], who used the Landauer transmission formula to obtain the quantumlimited conductivity. Following the same approach, we calculate the transmission probabilities of Dirac particles through a strip of graphene in the geometry of Fig. 1. [An earlier study of the same geometry counted the number of propagating modes, without determining their transmission probabilities [11].] The result depends on the aspect ratio $W / L$ of the strip and also on microscopic details of the upper and lower edge. For short and wide strips $(W / L \gg 1)$ these microscopic details become insignificant. For that reason we first discuss the simplest case of an edge which is smooth on the scale of the lattice spacing. This corresponds to confinement of the carriers by lattice straining. The opposite case of an abrupt edge (corresponding to confinement by etching) is considered later on.

The band structure of graphene has two valleys, which are decoupled in the case of a smooth edge. In a given valley the excitations have a two-component envelope wave function $\Psi=\left(\Psi_{1}, \Psi_{2}\right)$, varying on scales large com-

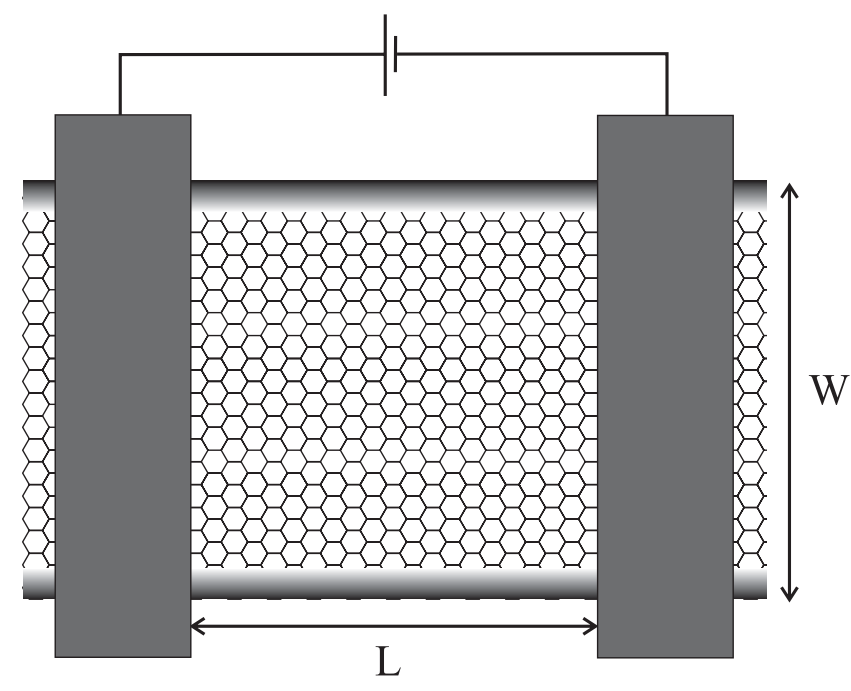

FIG. 1. Schematic of a strip of graphene of width $W$, contacted by two electrodes (black rectangles) at a distance $L$. A voltage source drives a current through the strip. A separate gate electrode (not shown) allows the carrier concentration in the strip to be tuned around the neutrality point. 
pared to the lattice spacing. This continuum description of the electronic states in graphene has been found to be quite accurate [12], and we will test it later on by comparing with a numerical solution of the scattering in a tight-binding model. The two components of $\Psi$ refer to the two sublattices in the two-dimensional honeycomb lattice of carbon atoms. (The additional spin degeneracy of the excitations does not play a role here.) The wave equation for $\Psi$ is the Dirac equation,

$$
\left[v p_{x} \sigma_{x}+v p_{y} \sigma_{y}+v^{2} M(y) \sigma_{z}+\mu(x)\right] \Psi(\boldsymbol{r})=\varepsilon \Psi(\boldsymbol{r}),
$$

with $v$ the velocity of the massless excitations of charge $e$ and energy $\varepsilon, \boldsymbol{p}=-i \hbar \partial / \partial \boldsymbol{r}$ the momentum operator, $\boldsymbol{r}=$ $(x, y)$ the position, and $\sigma_{i}$ a Pauli matrix. We choose the zero of energy such that the Fermi level is at $\varepsilon=0$.

The mass term $M(y)$ is zero in the interior of the strip and rises to $\infty$ at the edges $y=0$ and $y=W$, thereby confining the particles. As shown by Berry and Mondragon [13], infinite mass confinement corresponds to the boundary condition

$$
\left.\Psi_{1}\right|_{y=0}=\left.\Psi_{2}\right|_{y=0},\left.\quad \Psi_{1}\right|_{y=W}=-\left.\Psi_{2}\right|_{y=W} .
$$

As a result of this boundary condition, the transversal momenta are quantized as

$$
q_{n}=\frac{1}{W} \pi\left(n+\frac{1}{2}\right), \quad n=0,1,2, \ldots
$$

with $n$ labeling the modes. The quantization condition for Dirac particles confined by an infinite mass differs from the one for normal electrons confined by an infinite potential by the offset of $1 / 2$, originating from the $\pi$ phase shift in the boundary condition (2).

The electrostatic potential energy $\mu(x)=\mu$ for $0<x<$ $L$, varied by a gate voltage, determines the concentration of the carriers in the strip. The value $\mu=0$ corresponds to charge neutrality, being the point where electron and hole excitations are degenerate (known as the Dirac point). We model the electrodes by taking a large value $\mu(x)=\mu_{\infty}$ in the leads $x<0$ and $x>L$. (The parameter $\mu_{\infty}$ will drop out of the results if $\left|\mu_{\infty}\right| \gg|\mu|$.)

We calculate the transmission probabilities at the Fermi level by matching modes at $x=0$ and $x=L$. The matching condition for the Dirac equation is the continuity of the two components of $\Psi$. This ensures the conservation of the local current density $\boldsymbol{j}(\boldsymbol{r})=e \boldsymbol{v} \Psi^{\dagger}(\boldsymbol{r}) \cdot \boldsymbol{\sigma} \cdot \Psi(\boldsymbol{r})$, with $\boldsymbol{\sigma}=$ $\left(\sigma_{x}, \sigma_{y}\right)$, without requiring continuity of derivatives (as needed for the Schrödinger equation). There is a separate transmission probability $T_{n}$ for each of the $N$ propagating modes in the leads, because the matching condition does not mix the modes. [The integer $N \gg 1$ is given by $N=$ Int $\left(k_{\infty} W / \pi+\frac{1}{2}\right)$, with $\left|\mu_{\infty}\right|=\hbar v k_{\infty}$.]

Since the calculation is straightforward [14], we proceed directly to the results. At the Dirac point $\mu=0$ the transmission probability reads

$$
T_{n}=\frac{1}{\cosh ^{2} L q_{n}+\left(q_{n} / k_{\infty}\right)^{2} \sinh ^{2} L q_{n}} \rightarrow \frac{1}{\cosh ^{2}[\pi(n+1 / 2) L / W]} \quad \text { for } N \gg W / L .
$$

The formula (4) is essentially different from the textbook formula [15] for the transmission probability of nonrelativistic electrons through a potential barrier, which vanishes in the limit $N \rightarrow \infty$ at zero energy (relative to the top of the barrier).

The finite transmission probability at the Dirac point tends to the ballistic limit $T_{n} \rightarrow 1$ with increasing $|\mu|$. For $N \rightarrow \infty$ we find the expression [14]

$$
T_{n}=\left|\frac{k_{n}}{k_{n} \cos \left(k_{n} L\right)+i(\mu / \hbar v) \sin \left(k_{n} L\right)}\right|^{2},
$$

with $k_{n}=\sqrt{(\mu / \hbar v)^{2}-q_{n}^{2}}$.

The conductance $G$ and Fano factor $F$ follow by summing over the modes,

$$
G=g_{0} \sum_{n=0}^{N-1} T_{n}, \quad F=\frac{\sum_{n=0}^{N-1} T_{n}\left(1-T_{n}\right)}{\sum_{n=0}^{N-1} T_{n}},
$$

with $g_{0}=4 e^{2} / h$. (The factor 4 accounts for the spin and valley degeneracy.) The dependence of the conductivity $\sigma \equiv G \times L / W$ and the Fano factor at $\mu=0$ on the aspect ratio $W / L$ is plotted in Fig. 2 (solid curves). The dependence on $\mu$ at a fixed value of $W / L$ is shown in Fig. 3.
Figure 2 also contains results for a boundary condition corresponding to an abrupt edge (dashed curves). We considered a "metallic armchair" edge, in which the carbon lattice contains a multiple of three hexagons in the transverse direction, terminated at $y=0$ and $y=W$ by a horizontal bond. This edge mixes the valleys, so we need to consider a four-component wave function $\Psi=$ $\left(\Psi_{1}, \Psi_{2}, \Psi_{1}^{\prime}, \Psi_{2}^{\prime}\right)$. The first two components satisfy the Dirac equation (1), without the mass term, and the second two components satisfy the same equation with $p_{y} \rightarrow$ $-p_{y}$. The boundary condition is [12]

$$
\Psi_{1}=\Psi_{1}^{\prime}, \quad \Psi_{2}=\Psi_{2}^{\prime}, \quad \text { at } y=0, W .
$$

The valley degeneracy is broken for the lowest mode $(n=0)$, which is nondegenerate, while all higher modes $(n=1,2, \ldots)$ retain the twofold valley degeneracy (over and above the twofold spin degeneracy, common to all modes). For $\mu=0$ and $N \rightarrow \infty$ the transmission probabilities are given by

$$
T_{n}=\frac{1}{\cosh ^{2}(\pi n L / W)}, \quad n=0,1,2, \ldots
$$




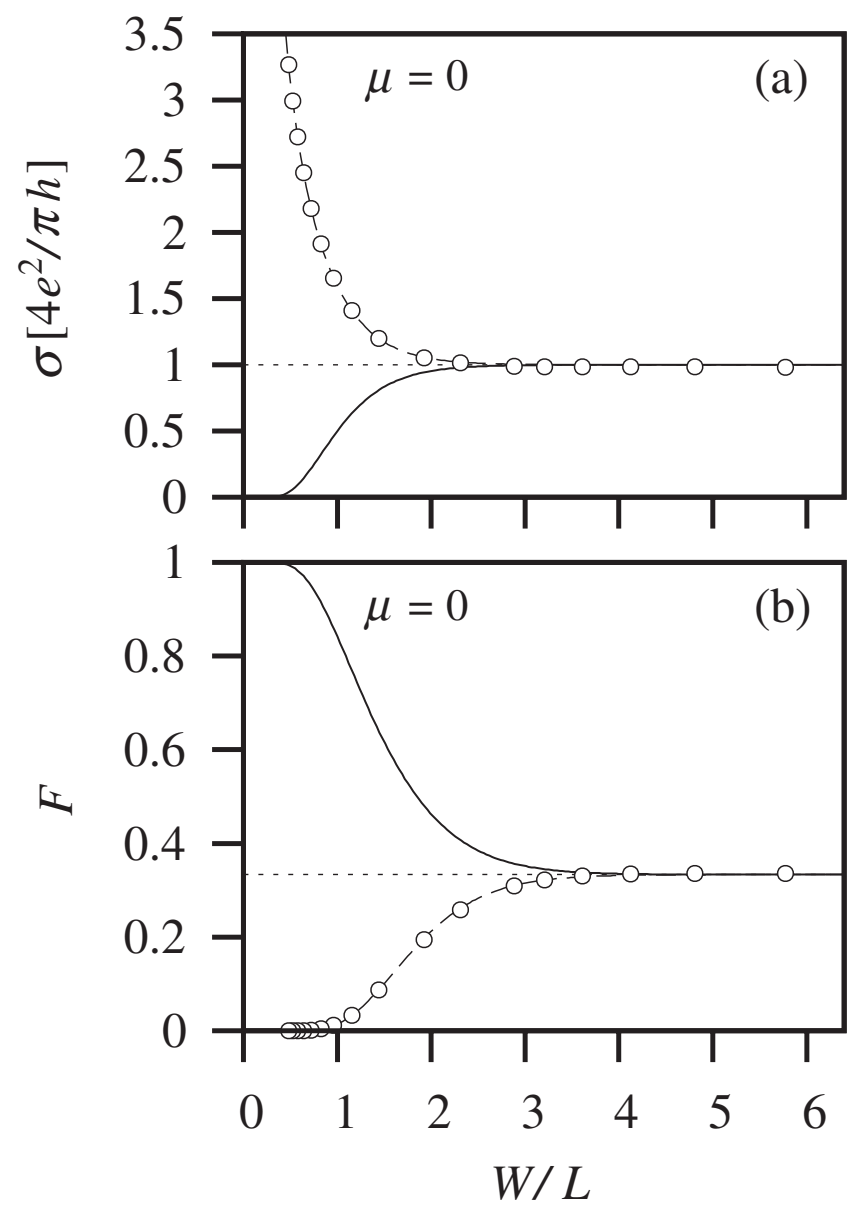

FIG. 2. Conductivity $\sigma \equiv G \times L / W$ and Fano factor $F$ at the Dirac point $(\mu=0)$, as a function of the aspect ratio of the graphene strip. The curves are calculated from Eq. (6) in the limit $N \rightarrow \infty$, for two different boundary conditions: smooth edge [solid curves, using Eq. (4)] and "metallic armchair" edge [dashed curves, using Eq. (8)]. The limit $W / L \rightarrow \infty$ (dotted lines) is given by Eq. (9), regardless of the boundary condition. The data points for the armchair edge are the result of a numerical solution of the tight-binding model on a hexagonal lattice.

The essential difference with the result (4) for the smooth edge is due to the absence of the $1 / 2$ offset in the quantization condition of the transverse momentum. The different boundary condition changes the strip from insulating to metallic in the limit $W / L \rightarrow 0$ [16], but has no effect in the opposite limit $W / L \rightarrow \infty$; cf. Fig. 2 .

To test the analytical results from the continuum description of graphene, we have also carried out numerical simulations using the tight-binding model with nearestneighbor hopping on a honeycomb lattice with metallic armchair edges. We took a total of $3 \times 10^{4}$ lattice sites for the graphene strip, coupling it to semi-infinite leads at the two ends. The valley degeneracy of modes $n=1,2, \ldots$ is now only approximate, but the relative magnitude of the mode splitting vanishes $\propto a / W$ as the width becomes large compared to the lattice spacing $a$ [17]. The numerical results, included in Fig. 2, are in excellent agreement with the analytical prediction.

Figure 3 shows that the minimum in the conductivity at the Dirac point is associated with a maximum in the Fano factor. The limiting behavior at the Dirac point for a short and wide strip is

$$
\sigma \rightarrow g_{0} / \pi, \quad F \rightarrow 1 / 3, \quad \text { for } W / L \rightarrow \infty .
$$

Note that these limits are already reached for moderate aspect ratios $W / L \gtrsim 4$. We derived this limiting behavior for two types of boundary conditions (smooth edge and metallic armchair edge), but we are confident that the result is universal, in the sense that it holds for the most general boundary condition at the edges of the graphene strip [as classified in Ref. [18] ].

The result (9) for the minimal conductivity agrees with other calculations [3-5], which start from an unbounded disordered system and then take the limit of infinite mean

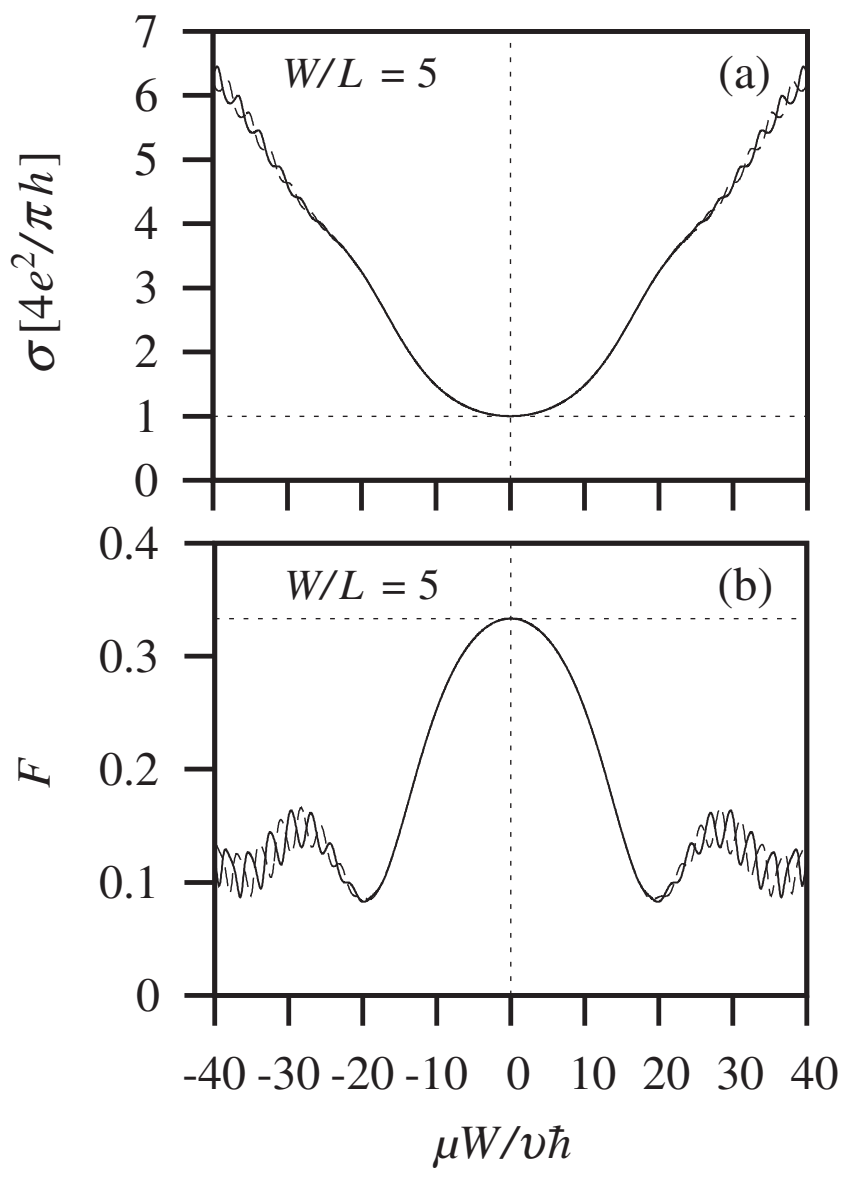

FIG. 3. Fermi energy dependence of the conductivity and Fano factor for a fixed aspect ratio. The conductivity minimum at the Dirac point corresponds to maximal Fano factor. The curves are calculated from Eq. (5) for the case of a smooth edge [solid curves, taking $\left.q_{n}=(n+1 / 2) \pi / W\right]$ or metallic armchair edge (dashed curves, taking $q_{n}=n \pi / W$ ). The oscillations signal the appearance of propagating modes in the graphene strip with increasing potential. 
free path $l$. There is no geometry dependence if the limits are taken in that order. In the ballistic bounded system considered here, a geometry dependence persists in the thermodynamic limit [6]. Existing experiments [1,2] are quasiballistic, with $l \simeq W<L$, finding $\sigma \approx g_{0}$. This would be consistent with Fig. 2(a) if the effective length in the experiment is set by $l$ rather than $L$. Residual disorder may also explain why the oscillations in Fig. 3(a), associated with the appearance of propagating modes, are not observed in the experimental gate voltage dependence.

The limit $F=1 / 3$ for the Fano factor is smaller than the value $F=1$ expected for a Poisson process. The same $1 / 3$ value appears in a disordered metal [7,8], where it is a consequence of classical diffusive dynamics. This correspondence is remarkable, since in our ideal graphene strip the classical dynamics is ballistic. The relativistic quantum dynamics of confined Dirac fermions is known to exhibit a jittering motion called "Zitterbewegung" [19], originating from the interference of states with positive and negative energy $[6,20]$. Our calculation implies that this relativistic quantum dynamics produces the same shot noise as classical diffusion.

In conclusion, we predict that electrical conduction through an ideal graphene strip is associated with timedependent current fluctuations - at zero temperature and without any impurities or lattice defects. The electrical noise is largest, relative to the mean current, when the Fermi energy is adjusted such that electrons and holes are degenerate. At this Dirac point the Fano factor (ratio of noise power and mean current) takes on the universal value $1 / 3$ for short and wide strips. Observation of this sub-Poissonian shot noise would be a unique demonstration of electrical noise produced by relativistic quantum dynamics.

We thank H. A. Fertig and A. Morpurgo for interesting discussions. This research was supported by the Dutch Science Foundation NWO/FOM and by the European Community's Marie Curie Research Training Network (Contract No. MRTN-CT-2003-504574, Fundamentals of Nanoelectronics). A. R. thanks the Polish Science Foundation for financial support.
[1] K. S. Novoselov, A. K. Geim, S. V. Morozov, D. Jiang, M. I. Katsnelson, I. V. Grigorieva, S. V. Dubonos, and A. A. Firsov, Nature (London) 438, 197 (2005).

[2] Y. Zhang, Y.-W. Tan, H. L. Stormer, and P. Kim, Nature (London) 438, 201 (2005).

[3] A. W. W. Ludwig, M.P. A. Fisher, R. Shankar, and G. Grinstein, Phys. Rev. B 50, 7526 (1994).

[4] K. Ziegler, Phys. Rev. Lett. 80, 3113 (1998).

[5] N. M. R. Peres, F. Guinea, and A.H. Castro Neto, Phys. Rev. B 73, 125411 (2006).

[6] M. I. Katsnelson, Eur. Phys. J. B 51, 157 (2006).

[7] C. W. J. Beenakker and M. Büttiker, Phys. Rev. B 46, 1889 (1992).

[8] K. E. Nagaev, Phys. Lett. A 169, 103 (1992).

[9] P.-E. Roche, M. Kociak, S. Guéron, A. Kasumov, B. Reulet, and H. Bouchiat, Eur. Phys. J. B 28, 217 (2002).

[10] E. Onac, F. Balestro, B. Trauzettel, C. F. J. Lodewijk, and L. P. Kouwenhoven, Phys. Rev. Lett. 96, 026803 (2006).

[11] N. M. R. Peres, A. H. Castro Neto, and F. Guinea, Phys. Rev. B 73, 195411 (2006).

[12] L. Brey and H. A. Fertig, cond-mat/0603107 [Phys. Rev. B (to be published)].

[13] M. V. Berry and R. J. Mondragon, Proc. R. Soc. Lond. A 412, 53 (1987).

[14] For details of the calculation of the transmission probability, see J. Tworzydło et al., cond-mat/0603315, Appendix A.

[15] The transmission probability of nonrelativistic electrons through a rectangular barrier at zero energy (relative to the top of the barrier) is given by $T_{n}=\left[\cosh ^{2} L q_{n}+\right.$ $\left.\left(k_{\infty}^{2} / 2 q_{n}^{2}-1\right)^{2}\left(k_{\infty}^{2} / q_{n}^{2}-1\right)^{-1} \sinh ^{2} L q_{n}\right]^{-1}$, with $q_{n}=$ $n \pi / W(n=1,2, \ldots)$. In contrast to the relativistic result (4), it vanishes $\propto\left(q_{n} / k_{\infty}\right)^{2}$ in the limit $k_{\infty} \rightarrow \infty$.

[16] For $W / L \rightarrow 0$ we find $G \rightarrow g_{0} / 2$ in the case of a metallic armchair edge. The factor $1 / 2$, due to the absence of valley degeneracy for the lowest mode, is missing from Ref. [11].

[17] The magnitude of the mode splitting follows from the formulas given in the appendix of K. Wakabayashi, M. Fujita, H. Ajiki, and M. Sigrist, Phys. Rev. B 59,8271 (1999).

[18] E. McCann and V. I. Fal'ko, J. Phys. Condens. Matter 16, 2371 (2004).

[19] E. Schrödinger, Sitzber. Preuß. Akad. Wiss. 24, 418 (1930).

[20] W. Zawadzki, cond-mat/0510184. 